\title{
Corrigendum
}

\section{A 3-D well-differentiated model of pediatric bronchial epithelium demonstrates unstimulated morphological differences between asthmatic and nonasthmatic cells}

Jeremy Parker, Severine Sarlang, Surendran Thavagnanam, Grace Williamson, Dara O'Donoghue, Remi Villenave, Ultan Power, Michael Shields, Liam Heaney and Grzegorz Skibinski

Pediatr Res 67: 17-22 (2010); doi:10.1203/PDR.0b013e3181c0b200

The authors recently learned that, as a result of erroneous information provided by a manufacturer, the concentration of epidermal growth factor added to the air-liquid interface (ALI) culture medium is incorrect as reported in the Methods section. In the paragraph headed "ALI cultures for establishment of well-differentiated mucociliary epithelium," the concentration of epidermal growth factor is given as $0.5 \mathrm{ng} / \mathrm{mL}$. The correct concentration, as subsequently confirmed by the manufacturer, is $10 \mathrm{ng} / \mathrm{ml}$. The error does not invalidate the authors' results. 